\title{
Multimodal Emotion Recognition in Response to Videos
}

\author{
Mohammad Soleymani, Member, IEEE, Maja Pantic, Fellow, IEEE, and \\ Thierry Pun, Member, IEEE
}

\begin{abstract}
This paper presents a user-independent emotion recognition method with the goal of recovering affective tags for videos using electroencephalogram (EEG), pupillary response and gaze distance. We first selected 20 video clips with extrinsic emotional content from movies and online resources. Then, EEG responses and eye gaze data were recorded from 24 participants while watching emotional video clips. Ground truth was defined based on the median arousal and valence scores given to clips in a preliminary study using an online questionnaire. Based on the participants' responses, three classes for each dimension were defined. The arousal classes were calm, medium aroused, and activated and the valence classes were unpleasant, neutral, and pleasant. One of the three affective labels of either valence or arousal was determined by classification of bodily responses. A one-participant-out cross validation was employed to investigate the classification performance in a user-independent approach. The best classification accuracies of 68.5 percent for three labels of valence and 76.4 percent for three labels of arousal were obtained using a modality fusion strategy and a support vector machine. The results over a population of 24 participants demonstrate that user-independent emotion recognition can outperform individual self-reports for arousal assessments and do not underperform for valence assessments.
\end{abstract}

Index Terms-Emotion recognition, EEG, pupillary reflex, pattern classification, affective computing.

\section{INTRODUCTION}

$\mathrm{E}$ MOTIONS play an important role in viewers' content selection and consumption. With the rapid expansion of digital multimedia content, alternative methods to the existing explicit tagging are needed to enrich the pool of tagged content. When a user watches video clips or listens to music, he/she may experience certain feelings and emotions [1], [2], [3] which manifest through bodily and physiological cues, e.g., pupil dilation and contraction, facial expressions, e.g., frowning, and changes in vocal features, e.g., laughter.

In order to translate a user's bodily and behavioral reactions to emotions, reliable emotion assessment techniques are required. Emotion assessment is a challenging task; even users are not always able to express their emotion with words and the emotion self-reporting error is not negligible. This makes it difficult to define a ground truth. Affective self-reports might be held in doubt because users cannot always remember all the different emotions they had felt during watching a video and/or might misrepresent their feelings due to self-presentation, e.g., a user wants to

- M. Soleymani and T. Pun are with the Computer Vision and Multimedia Laboratory, Computer Science Department, University of Geneva, Battelle Campus, Building A, Rte. de Drize 7, Carouge (GE) CH-1227, Switzerland. E-mail: \{mohammad.soleymani, thierry.pun\}@unige.ch.

- M. Pantic is with the Department of Computing, Imperial College London, 180 Queen's Gate, London SW7 2AZ, United Kingdom, and the Faculty of Electrical Engineering, Mathematics and Computer Science, University of Twente, Drienerlolaan 5, 7522 NB Enschede, The Netherlands.

E-mail:m.pantic@imperial.ac.uk, panticm@cs.utwente.nl.

Manuscript received 31 Jan. 2011; revised 14 Oct. 2011; accepted 21 Nov. 2011; published online 29 Nov. 2011.

Recommended for acceptance by E. Andre.

For information on obtaining reprints of this article, please send e-mail to: taffc@computer.org, and reference IEEECS Log Number

TAFFC-2011-01-0008.

Digital Object Identifier no. 10.1109/T-AFFC.2011.37. show he is courageous whereas in reality he was scared [4] The emotion recognition system provides us with an alternative that reduces the effort of deciding on the right label and on defining the right questions or methods to assess emotions explicitly.

One of the most accepted and well-known theories which explains the process of emotional experience is appraisal theory. According to this theory, cognitive judgment or appraisal of situation is a key factor in the emergence of emotions [5], [6], [7]. According to Orthoney, Clore, and Collins (OCC) [6], emotions are experienced with the following scenario: First, there is a perception of an event, object, or an action. Then, there will be an evaluation of events, objects, or actions according to personal wishes or norms. Finally, the perception and evaluation result in a specific emotion. Considering this scenario for an emotional experience in response to multimedia content, emotions arise first through sympathy with the presented emotions in the content. During the appraisal process for an emotional experience in response to multimedia content, a viewer examines events, situations, and objects with respect to their novelty, pleasantness, goal, attainability, copability, and compatibility with his/her norms. Then, the viewer's perception induces specific emotions which change the viewer's physiological responses, motor actions, and feelings [8].

Scherer [9] categorized emotions into utilitarian and aesthetic emotions. Emotional responses to videos are a mixture of both utilitarian and aesthetic emotions with an emphasis on the later one. Existence of aesthetic emotional responses discourages simply using six well-known basic emotions in the context of emotion understanding of videos.

\subsection{Background}

Emotional responses to multimedia content have been studied from three perspectives. There has been a research 
trend toward estimating emotions from multimedia content [10], [11], [12]. On the other hand, recognizing emotions induced by videos has been studied in the affective computing community [13], [14], [15], [3], [16]. The emotion recognition has also been used in applications such as detecting topical relevance or summarizing videos [17], [13], [18]. This paper presents an emotion recognition method using EEG signals and eye gaze data in response to videos using users' bodily responses.

Hanjalic and $\mathrm{Xu}$ [10] introduced "personalized content delivery" as a valuable tool in affective indexing and retrieval systems. In order to represent affect in video, they first selected video and audio content-based features based on their relation to the valence-arousal space that was defined as an affect model. Irie et al. [19] proposed a latent topic model by defining affective audio-visual words in the content of movies to detect emotions in movie scenes. They extracted emotion category-specific audio-visual features named affective audio-visual words. These higher level features were used to classify movie scenes using a latent topic driving model. This model takes into account temporal information which is the effect of the emotion from precedent scene to improve affect classification. The probability of emotional changes between consecutive scenes was also used in [20] to improve the emotional classification of movie scenes using content features.

Emotional characteristics of videos have also improved music and image recommendation. Shan et al. [12] used affective characterization using content analysis to improve film music recommendation. Tkalčič et al. showed how affective information can improve image recommendation [11]. In their image recommendation scenario, affective scores of images from the international affective picture system (IAPS) [21] were used as features for an image recommender. They conducted an experiment with 52 participants to study the effect of using affective scores. The image recommender using affective scores showed a significant improvement in the performance of their image recommendation system.

Joho et al. [17], [13] developed a video summarization tool using facial expressions. A probabilistic emotion recognition based on facial expressions was employed to detect emotions of 10 participants watching eight video clips. The participants were asked to mark the highlights of the video with an annotation tool after the experiments. The expression change rate between different emotional expressions and the "pronounce level" or amount of expression were used as features to detect personal highlights in the videos. The pronounce levels they used ranged from highly expressive emotions, surprise and happiness, to no expression or neutral. They have also extracted two affective content-based features which were audio energy and visual change rate from videos to create an affective curve in the same way as the affective highlighting method proposed by Hanjalic and $\mathrm{Xu}[10]$.

There is long-standing research on emotion assessment from physiological signals [1], [22], [23], [24], [14], [25]. Among these studies, few of them achieved notable results using video stimuli. Lisetti and Nasoz used physiological response to recognize emotion in response to movie scenes
[14]. The movie scenes elicited six emotions, namely, sadness, amusement, fear, anger, frustration, and surprise. They achieved a high recognition rate of 84 percent for the recognition of these six emotions. However, the classification was based on the analysis of the signals in response to preselected segments in the shown video known to be related to highly emotional events.

Takahashi [15] recorded EEG and peripheral physiological signals from 12 participants. He then classified the responses to emotional videos into five classes, namely, joy, sadness, disgust, fear, and relax. He achieved the accuracy of 41.7 percent using EEG signals. However, the feature level fusion (FLF) of EEG signals and peripheral physiological signals failed to improve the classification accuracy.

An affective characterization for movie scenes using peripheral physiological signals as well as multimedia content features was proposed by Soleymani et al. [3]. Eight participants watched 64 movie scenes and selfreported their emotions. Affective correlates between different physiological and content features were studied. A linear regression trained by relevance vector machines (RVM) was utilized to estimate each clip's affect from physiological and content features.

Koelstra et al. [16] recorded EEG and peripheral physiological signals of six participants in response to music videos. Participants rated their felt emotions by means of arousal, valence, and like/dislike rating. The emotional responses of each participant were classified into two classes of low/high arousal, low/high like/dislike, and low/high valence. The average classification rates varied between 55 and 58 percent which is slightly above random level.

In a more recent study, Kolodyazhniy et al. [26] used peripheral physiological signals to recognize neutral, fear, and sadness responses to movie excerpts. During the presentation of videos to the participants, they introduced startle stimuli using randomly generated white noise sounds to boost physiological responses. Their system was able to recognize sadness, fear, and neutral emotional states with the recognition rate of 77.5 percent in a participantindependent approach.

Eye gaze and pupillary responses have been used extensively to measure attention. However, we are not aware of research on how emotions affect eye gaze while watching videos; therefore, the eye gaze itself has not been used for emotion recognition. The pupillary response is the measurement of pupil diameter over time. Pupils can dilate or constrict in response to light, cognitive, attentional, and emotional stimuli [27], [28]. Gao et al. [29] showed the significance of using pupillary reflex for emotion assessment after reducing the light effect using a real-time feedback.

\subsection{Potential Application}

Characterizing multimedia content with relevant, reliable, and discriminating tags is vital for multimedia information retrieval. Affective characteristics of multimedia are important features for describing multimedia content and can be presented by such emotional tags. Implicit affective tagging refers to the effortless generation of subjective and/ or emotional tags. Implicit tagging of videos using affective information can help recommendation and retrieval systems to improve their performance [12], [11], [30]. 


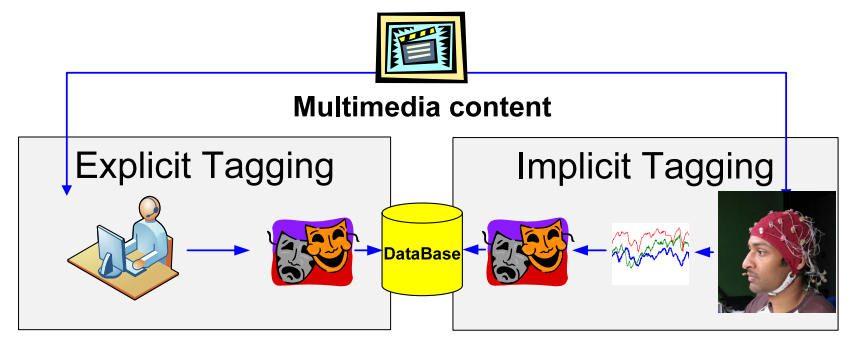

Fig. 1. Implicit affective tagging versus explicit tagging scenarios. The analysis of the bodily responses replaces the direct interaction between user and the computer. Therefore, users do not have to be distracted for tagging the content.

Currently, social media websites encourage users to tag their content. However, the users' intent when tagging multimedia content does not always match the information retrieval goals. A large portion of user-defined tags are either motivated by the goal of increasing the popularity and reputation of a user in an online community or based on individual and egoistic judgments [31]. Implicit tagging does not interrupt users while listening or watching a video. Moreover, in the presence of a reliable implicit tagging measurement method, determined tags carry less irrelevant and inaccurate information.

Users do not evaluate media content on the same criteria. Some might tag multimedia content with words to express their emotion while others might use tags to describe the content. For example, a picture receives different tags based on the objects in the image, the camera by which the picture was taken, or the emotion a user felt looking at the picture. Scherer defines this by intrinsic and extrinsic appraisal [9]. Intrinsic appraisal is independent from the current goals and values of the viewer while extrinsic or transactional appraisal leads to feeling emotions in response to the stimuli. For example, the content's intrinsic emotion of a picture with a smiling face is happiness whereas this person might be a hatred figure to the viewer and the extrinsic appraisal leads to unpleasant emotions. What we want to detect is the latter one that is the emotion felt by the viewer.

The goal of implicit affective tagging is thus to monitor the reactions of a person in response to a particular multimedia content and automatically recognize the corresponding tag. These responses can be used to reliably generate affective tags. A scheme of implicit tagging scenario versus explicit tagging is shown in Fig. 1.

In the proposed implicit tagging scenario, multimedia content will be tagged based on the bodily reactions of users recorded by a physiological acquisition device and an eye gaze tracker. The reactions can be used both to find tags common to a population and to develop a personal profile possibly in combination with user preferences and browsing history. With recently marketed physiological devices such as Neurosky ${ }^{1}$ and Emotiv helmet, ${ }^{2}$ physiological interfaces are likely going to be the emerging humancomputer interfaces of the future.

\subsection{Research Questions}

Although individual differences are always present in emotional reactions, there usually also exists one more

1. http://www.neurosky.com/.

2. http://www.emotiv.com/. common affective response in a population to a multimedia content. For example, the scenes from a drama movie induce sadness in most of the people. For a dramatic scene, sadness can be considered the popular or frequently felt emotion. In this paper, we investigate and show the feasibility and comparable performance of a user-independent emotion recognition to detect the dominant or commonly selected affective tags. The implicit tagging application limited our choices for modalities to cues which are measurable while participants are sitting and are mostly in passive mode. The research questions that this paper investigates are:

1. Is it possible to design an accurate and userindependent classification protocol to recognize emotions from pupillary reflex, EEG signals, and other bodily responses in response to video content?

2. Can nonverbal affective cues replace affective selfreport with comparable emotion recognition performance and no requisite of direct user inputs?

The rest of the paper is organized as follows: The emotion model, video data set, experimental protocol, and physiological data set classification are discussed in Section 2. Section 3 presents and discusses the experimental results and compares their performance to self-reports. The current methods' open issues and future work are discussed in Section 4. The paper is concluded in Section 5.

\section{Material and Methods}

\subsection{Emotional Model}

Although the most straightforward way to represent an emotion is to use discrete labels such as fear and joy, labelbased representations have some disadvantages, the main one being that labels are not cross lingual: Emotions do not have exact translations in different languages, e.g., "disgust" does not have an exact translation in Polish [32]. Psychologists therefore often represent emotions or feelings in an n-dimensional space (generally two or three-dimensional). The most famous such space, which is used in the present study and originates from cognitive theory, is the $2 \mathrm{D}$ valence-arousal or pleasure-arousal space [33]. The valence scale ranges from unpleasant to pleasant. The arousal scale ranges from passive to active or excited.

\subsection{Preliminary Study}

In the preliminary study, 21 commercially produced movies were first segmented into their scenes. Scenes longer than 2 minutes were divided into shorter 2 minute long excerpts. From these excerpts, 155 emotional video clips containing full or part of movie scenes were manually selected. The 155 selected videos were shown to more than 50 participants; each video clip received 10 annotations on average [34]. The preliminary study was conducted utilizing an online affective annotation system in which the participants were able to use a web interface to report their emotions in response to the videos played by a web-based video player (see Fig. 2). In the case of using videos from online repositories, the full length videos were used in the data set.

In the preliminary study, the participants were thus asked to self-assess their emotion by reporting the felt arousal (ranging from calm to excited/activated) and 


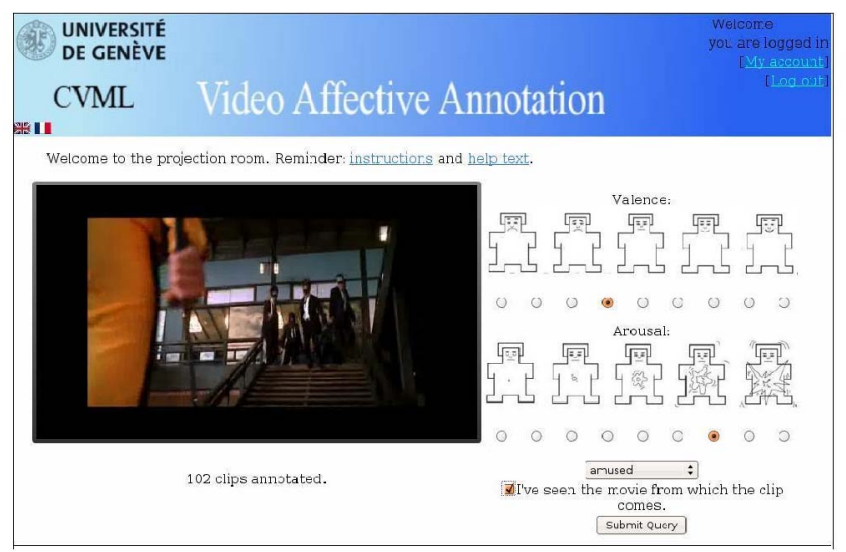

Fig. 2. A snapshot of the online affective annotation system. Arousal and valence were assessed using SAM manikins. Participants were able to give their emotional tag using a drop down menu.

valence (ranging from unpleasant to pleasant) on nine point scales as well as emotional keywords. Fourteen video clips were chosen based on the preliminary study from the clips which received the highest number of emotional keyword tags in different emotion categories, which are listed in the Table 1. Videos were selected to cover different emotional responses (see Fig. 3). Three other popular video clips from online resources were added to this set (two for joy/ happiness and one for disgust). Three past weather forecast reports (retrieved from youtube.com) were also used as neutral emotion clips. The videos from online resources

TABLE 1

The Video Clips and Their Sources

\begin{tabular}{l|l|l}
\hline \hline Code & $\begin{array}{l}\text { Emotion } \\
\text { Labels }\end{array}$ & Video clips sources \\
\hline $\mathbf{1}$ & Act., Unp. & Hannibal \\
\hline $\mathbf{2}$ & Act., Unp. & The Pianist \\
\hline $\mathbf{3}$ & Med., Pls. & Mr. Bean's holiday \\
\hline $\mathbf{4}$ & Act., Neu. & Ear worm (blip.tv) \\
\hline $\mathbf{5}$ & Med., Neu. & Kill Bill VOL I \\
\hline $\mathbf{6}$ & Med., Pls. & Love actually \\
\hline $\mathbf{7}$ & Med., Pls. & Mr. Bean's holiday \\
\hline $\mathbf{8}$ & Cal., Pls. & The thin red line \\
\hline $\mathbf{9}$ & Med., Neu. & The shining \\
\hline $\mathbf{1 0}$ & Med., Pls. & Love actually \\
\hline $\mathbf{1 1}$ & Act., Unp. & The shining \\
\hline $\mathbf{1 2}$ & Med., Unp. & Gangs of New York \\
\hline $\mathbf{1 3}$ & Act., Unp. & Silent hill \\
\hline $\mathbf{1 4}$ & Med., Unp. & The thin red line \\
\hline $\mathbf{1 5}$ & Cal., Neu. & $\begin{array}{l}\text { AccuWeather New York weather report } \\
\text { (youtube.com) }\end{array}$ \\
\hline $\mathbf{1 6}$ & Act., Unp. & American history X \\
\hline $\mathbf{1 7}$ & Cal., Neu. & $\begin{array}{l}\text { AccuWeather Detroit weather report } \\
\text { (youtube.com) }\end{array}$ \\
\hline $\mathbf{1 8}$ & Act., Pls. & Funny cats (youtube.com) \\
\hline $\mathbf{1 9}$ & Cal., Neu. & $\begin{array}{l}\text { AccuWeather Dallas weather } \\
\text { (youtube.com) }\end{array}$ \\
\hline $\mathbf{2 0}$ & Act., Pls. & Funny (blip.tv) \\
\hline $\mathbf{9 m o t}$ & \\
\hline
\end{tabular}

The emotion labels are: calm (Cal.), medium aroused (Med.), activated (Act.), unpleasant (Unp.), neutral valence (Neu.), Pleasant (Pls.).

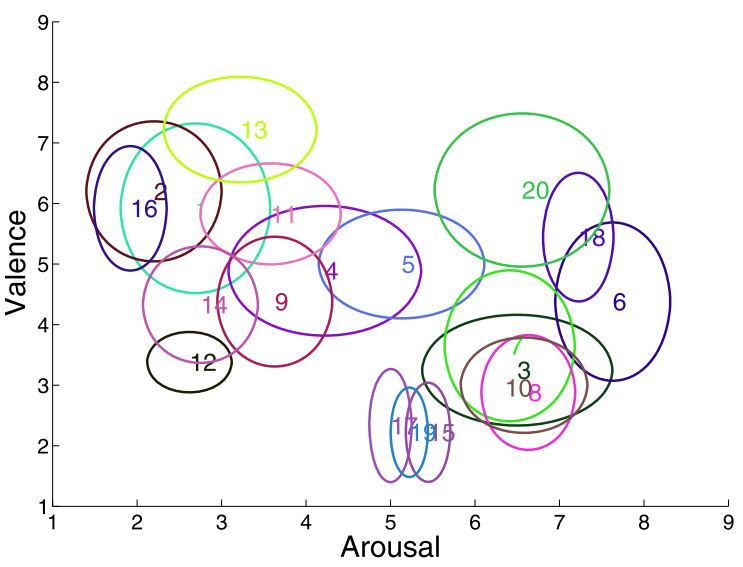

Fig. 3. Stimulus videos are shown in the valence-arousal plane. The center of the ellipses represents the mean arousal and valence and the horizontal and vertical radius represents the standard deviation of the online assessments. The clip codes are printed at the center of each ellipse.

were added to the data set to enable us to distribute some of the emotional video samples with the recorded multimodal data set described below. Table 1 gives the emotional labels, titles, and sources of the emotional video clips.

The median arousal and valence was used to determine ground truth labels with the following procedure. First, the values assessed by the online questionnaire were centered and then three equal length intervals were defined on the assessment range (arousal, valence $\in[1,9]$ ). The labels assigned to all videos are given in Table 1 . The distribution of online self-emotions for the selected videos is shown in Fig. 3.

Ultimately, 20 videos were selected to be shown which were between 34.9 to $117 \mathrm{~s}$ long $(M=81.4 \mathrm{~s}, \mathrm{SD}=22.5 \mathrm{~s})$. Psychologists recommended videos from 1 to 10 minutes long for elicitation of a single emotion [35], [2]. Here, the video clips were kept as short as possible to avoid multiple emotions or habituation to the stimuli while keeping them long enough to observe the effect.

\subsection{Experiment Protocol and Setup}

A multimodal recording setup was arranged to record facial videos, audio and vocal expressions, eye gaze, and physiological signals simultaneously (see Fig. 4). The experiment was controlled by the Tobii studio software (http://www.tobii.com). In order to synchronize different modalities, device generated time stamps were recorded along with audio and physiological signals. These time stamps consisted of time series with square shaped periodic signal $(60 \mathrm{~Hz})$ representing the moments when the cameras' shutters were open to capture each frame. The synchronization method and hardware setup details are given in Lichtenauer et al. [36].

The Biosemi active II system ${ }^{3}$ with active electrodes was used for physiological signals acquisition. Physiological signals including ECG, EEG (32 channels), galvanic skin response (GSR), respiration amplitude, and skin temperature were recorded while the videos were shown to the participants. Peripheral physiological signals, facial videos, and vocal expressions modalities were not employed in this

3. http://www.biosemi.com. 


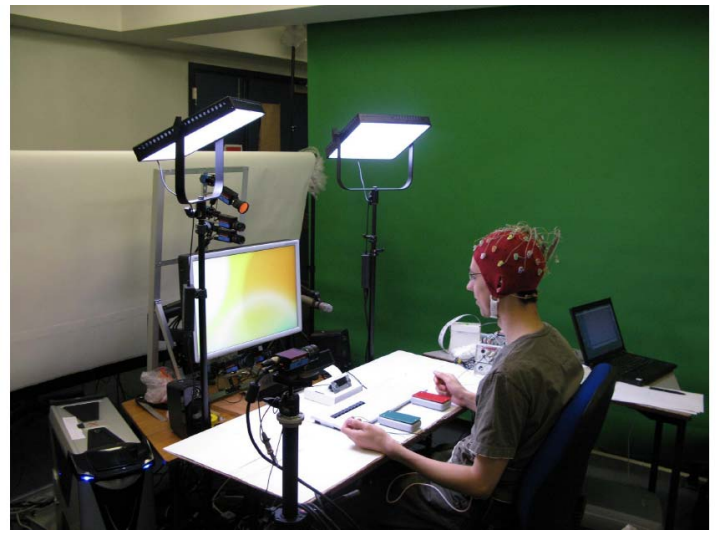

Fig. 4. The experimental setup. Six video cameras were recording facial expressions during the experiment. The modified keyboard is visible in front of the participant.

paper; therefore, all the results of this paper are only based on EEG signals, pupillary response, and gaze distance recorded by eye gaze tracker. However, the classification protocol presented in this paper can be applied to a wide variety of modalities and is not limited to the utilized modalities.

Participants were asked to report their felt emotions by indicating their felt arousal and valence on a nine point scale. To simplify the interface, a keyboard was provided with only nine numerical keys and the participant could answer each question by pressing one of the nine.

Thirty participants with different cultural and educational backgrounds volunteered to participate in response to a campus wide call for volunteers at Imperial College London. Out of the 30 young healthy adult participants, 17 were female and 13 were male; ages varied between 19 to 40 years old $(M=26.06, S D=4.39)$. Participants had different educational background from undergraduate students to postdocs with different English proficiency from intermediate to native speakers. The data recorded from six participants were not analyzed due to technical problems, poor signal quality, and unfinished data collection. Hence, the analysis results of this paper are only based on the responses recorded from 24 participants. The results in Section 3 of this paper are only based on eye gaze data and EEG signals. This database is freely available to the academic community and is easily accessible through a web-interface. ${ }^{4}$

The participants were informed about the experiment and their rights with a verbal introduction, by e-mail, and through a consent form. Participants were trained about the interface before the experiment and during the setup time. The participants were also introduced to the meaning of arousal, valence in the self-assessment procedure, and to the nature of the video content.

In emotional-affective experiments, the bias from the emotional state needs to be removed. For this purpose, before each emotional video, a short neutral clip randomly selected from the clips provided by the Stanford psychophysiology laboratory [2] was shown to the participants.

Each trial started with a short neutral clip. After watching the short neutral clip, one of the 20 video clips

4. http://mahnob-db.eu.

\begin{tabular}{|c|c|c|c|c|}
\hline Trial 1 & Trial 10 & Trial 11 & & Trial 20 \\
\hline Neutral clip & Emotional clip & $\begin{array}{c}\text { Self } \\
\text { assessment }\end{array}$ \\
\hline 15 seconds & \multicolumn{1}{|c|}{ 1 2 minutes } & time \\
\hline
\end{tabular}

Fig. 5 . Each trial started by a $15 \mathrm{~s}$ neutral clip and continued by playing one emotional clip. The self-assessment was done at the end of each trial. There were 20 trials in each session of the experiment.

was played. Video clips were played from the data set in random order. After watching the video clip, the participant filled in the self-assessment form, which appeared automatically. In total, the time interval between the start of a trial and the end of the self-reporting phase was approximately two and half minutes. This interval included playing the neutral clip, playing the emotional clip, performing the self-assessment. Running of the whole protocol took, on average, 50 minutes, in addition to 30 minutes setup time (see Fig. 5).

\subsection{Preprocessing and Feature Extraction}

\subsubsection{EEG Signals}

Psychological studies regarding the relations between emotions and the brain are uncovering the strong implication of cognitive processes in emotions [37], [38]. As a result, EEG signals carry valuable information about the participants' felt emotions.

Electroencephalogram signals were recorded with a $1,024 \mathrm{~Hz}$ sampling rate and later downsampled to $256 \mathrm{~Hz}$ to reduce the memory and processing costs. EEG signals were recorded using active $\mathrm{AgCl}$ electrodes placed according to the international 10-20 system. The layout of EEG electrodes on the cap is shown in Fig. 6. The unwanted artifacts, trend, and noise were reduced prior to extracting the features from EEG data by preprocessing the signals. Drift and noise reduction were done by applying a $4-45 \mathrm{~Hz}$ band-pass filter. Other artifacts, such as muscular activity, were kept at minimum level by instructing the participants

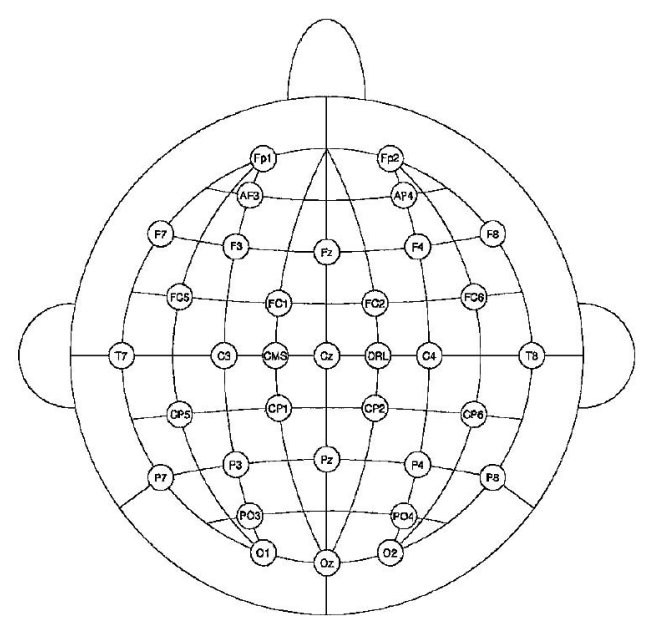

Fig. 6. The EEG cap layout for 32 EEG in addition to two reference electrodes. Retrieved from Biosemi website (http://www.biosemi.com). 
TABLE 2

All the Features Extracted from Eye Gaze Data and EEG Signals

\begin{tabular}{ll}
\hline \hline Eye gaze data & Extracted features \\
\hline Pupil diameter & $\begin{array}{l}\text { standard deviation, spectral power in the fol- } \\
\text { lowing bands: ]0, 0.2]Hz, ]0.2, 0.4] } \mathrm{Hz}, \quad] 0.4, \\
0,6] \mathrm{Hz} \text { and }] 0.6,1] \mathrm{Hz}\end{array}$ \\
approach time ratio, avoidance time ratio, ap- \\
proach rate \\
Eye blinking & $\begin{array}{l}\text { blink depth, blinking rate, length of the longest } \\
\text { blink, time spent with eyes closed } \\
\text { theta, slow alpha, alpha, beta, and gamma PSD } \\
\text { for each electrode. The spectral power asymme- } \\
\text { try between 14 pairs of electrodes in the four } \\
\text { bands of alpha, beta, theta and gamma. }\end{array}$ \\
\hline
\end{tabular}

to minimize their movements while videos were playing. Biosemi active electrodes record EEG signals referenced to common mode sense electrode (CMS) as a part of its feedback loop. In order to gain the full common-mode rejection ratio (CMRR) at $50 \mathrm{~Hz}$, EEG signals should be rereferenced to another reference. EEG signals were thus rereferenced to the average reference to maximize signal-tonoise ratio.

The spectral power of EEG signals in different bands was found to be correlated with emotions [39], [40], [25]. Power spectral density (PSD) from different bands were computed using fast Fourier transform (FFT) and Welch algorithm [41]. In this method, the signal is split into overlapping segments and the PSD is estimated by averaging the periodograms. The averaging of periodograms results in smoother power spectrum. The PSD of each electrode's EEG signals was estimated using $15 \mathrm{~s}$ long windows with 50 percent overlapping.

The logarithms of the PSD from theta $(4 \mathrm{~Hz}<\mathrm{f}<8 \mathrm{~Hz})$, slow alpha $(8 \mathrm{~Hz}<\mathrm{f}<10 \mathrm{~Hz})$, alpha $(8 \mathrm{~Hz}<\mathrm{f}<12 \mathrm{~Hz})$, beta $(12 \mathrm{~Hz}<\mathrm{f}<30 \mathrm{~Hz})$, and gamma $(30 \mathrm{~Hz}<\mathrm{f})$ bands were extracted from all 32 electrodes as features. In addition to power spectral features, the difference between the spectral power of all the 14 symmetrical pairs on the right and left hemisphere was extracted to measure the possible asymmetry in the brain activities due to the valence of an emotional stimuli [42], [39]. The asymmetry features were extracted from all mentioned bands except slow alpha. The total number of EEG features of a trial for 32 electrodes is $14 \times 4+32 \times 5=216$ features. A list of extracted EEG features is given in Table 2.

\subsubsection{Eye Gaze Data}

The $\mathrm{X} 120$ Tobii $^{5}$ eye gaze tracker provides the position of the projected eye gaze on the screen, the pupil diameter, the moments when the eyes were closed, and the instantaneous distance of the participant's eyes to the gaze tracker device positioned below the screen. The eye gaze data were sampled at $60 \mathrm{~Hz}$. The blinking moments are also extractable from eye gaze data. The eye gaze itself is highly dependent on the content and therefore it was not used directly for emotion

5. http://www.tobii.com. recognition. However, pupil diameter has been shown to change in different emotional states [27], [28].

A linear interpolation was used to replace the missing pupil diameter samples due to eye blinking. Then, the average diameter of right and left eye pupil was used as the pupil diameter time series. The major cause of pupil diameter variation comes from lighting; therefore, the participants' responses to the same video (stimuli) in the controlled lighting environment follow similar patterns. There are different parametric models for pupillary light reflex [43], [44]. However, these parametric models are not error free and calculating their numerous parameters is rather difficult without specific light reflex experiment. It has been shown that the pupillary light reflex magnitude changes with age and between different people [43]. Most of the participants in our experiment were young, in their 20s; therefore, the aging effect was assumed to be negligible. The difference between the magnitudes can be reduced by normalizing the pupil diameter time series. Consequently, we extracted the light reflex using a nonparametric estimation from the data. This common lighting reflex pattern was estimated for each video using principal component analysis (PCA).

If $Y$ is the $M \times N_{p}$ matrix containing the centered and normalized pupillary responses to the same video from $N_{p}$ participants and $M$ samples, then $Y$ consists of three components:

$$
Y=X+Z+E .
$$

$X$ is the lighting response which is the strongest effect in the signal. $Z$ is the parasympathetic emotional and attentional response and $E$ is the noise originated from measurement. These three components originated from independent sources and the decorrelating characteristic of principal component analysis is able to separate these three. First, $Y$ was decomposed using principal component analysis into $N_{p}$ components. The first principal component is assumed to be a close estimation of the lighting reflex. The normalized principal component was then removed from normalized time series. Then, the remaining residual part includes $Z+E$ :

$$
\begin{gathered}
Y=U D V^{T}, \\
A_{p}=U D, \\
S_{p}=V^{T}, \\
Y_{1}=A_{p 1} S_{p 1}, \\
Y_{R}=Y-Y_{1} .
\end{gathered}
$$

If we decompose $Y$ using singular value decomposition (SVD), $U$ is a matrix with eigen vectors of $Y Y^{T}$ as its column. $D$ is a diagonal matrix whose diagonal values are the eigen values of $Y Y^{T}$. Finally, the columns of $V$ are the eigen vectors of $Y^{T} Y$ (see (2)). From the principal components of $Y, A_{p}$ we can reconstruct the first principal component or the light reflex pattern $Y_{1}$ (see (3)). To remove the light reflex component, $Y_{1}$, from all the time series, it is enough to subtract it from the original data (see (5) and (6)). 
$Y_{R}$ is the residual part which contains the emotional and attentional pattern in addition to the noise.

After removing the linear trend, the power spectrum of the pupil diameter variation was computed. Standard deviation and spectral features were extracted from the pupil diameter. The Hippus effect is the small oscillations of eye pupil diameter between 0.05 to $0.3 \mathrm{~Hz}$ and with the amplitude of $1 \mathrm{~mm}$ [43], [45]. Hippus effect has been shown to be present when one is relaxed or passive. In the presence of mental activity, the effect will disappear. The Hippus effect is extracted by the first two power spectral features which are covering up to $0.4 \mathrm{~Hz}$. The rate of eye blinking is shown to be correlated with anxiety [46]. From the eye blinks, the eye blinking rate, the average, and maximum blink duration were extracted as features. In addition to the eye blinking features, the amount of time a participant spent with his/her eyes closed was also used as a feature to detect possible eye closing due to unpleasant emotions.

Although the participants were asked not to move during the experiment, there were small head movements which manifested themselves in the distance between participants' eyes and the eye gaze tracker. The distance of the participant to the screen and its changes provide valuable information about the participants' posture. The total change in the distance of the user to the gaze tracker, gaze distance, was calculated to measure the possible approach and avoid phenomena. The amount of time the participant spent per trial getting close or far from the screen was computed as well. These features were named approach and avoidance ratio to represent the amount of time each participant spent getting close to or going far from the screen. The frequency of the participants' movement toward the screen during each trial, approach rate, was also extracted. Ultimately, 12 features were extracted from the eye gaze data. A summary of all extracted features is given in Table 2 .

\subsubsection{Feature Normalization}

All the extracted features were numerical. To reduce the between participant differences, it is necessary to normalize the features. Maximum-Minimum normalization was applied on each feature of the features set separately on each participant's signals. In this normalization, the minimum value for any given feature is subtracted from the same feature of a participant and the results were divided by the difference between the maximum and minimum values.

\subsection{Emotion Classification}

With the proposed interparticipant emotion recognition, the goal is to find the emotional class with the highest agreement within a population. The most popular emotional class or tag can satisfy a larger population of viewers in a video retrieval scenario. For each video from the data set, the ground truth was thus defined by computing the median of arousal and valence scores given on a nine point scale. The median values were then categorized into three classes with equal intervals. According to this definition, we can name these classes calm, medium aroused, and activated for arousal and unpleasant, neutral, and pleasant for valence.

An SVM classifier with RBF kernel was employed to classify the samples using features from each of the two modalities. Prior to classification, a feature selection was used to select discriminative features as follows: First, a one-way ANOVA test was done on only the training set for each feature with the class as the independent variable. Then, any feature for which the ANOVA test was not significant $(p>0.05)$ was rejected. This feature selection criterion was hence recalculated for each cross-validation's iteration. A leave-one-participant-out cross-validation technique was used to validate the user-independent classification performance. At each step of cross validation, the samples of one participant were taken out as a test set and the classifier was trained on the samples from the rest of the participants. This cross validation was employed to imitate the effect of introducing a new user to our emotion recognition system. This process was repeated for all participants' data.

\subsubsection{Modality Fusion Strategy}

Classification in different modalities can be fused at both the feature level and the decision level. We applied these two fusion strategies and reported their results. With the feature level fusion, the feature vectors from different modalities were concatenated to form a larger feature vector. The feature selection and classification methods were then applied to the new feature set. However, with the decision level fusion (DLF), classification was performed on each modality separately and the classification outcomes were fused to generate the fusion results. In [15], feature level fusion of EEG and peripheral physiological signals did not improve the single modality results. On the other hand, Chanel et al. [25] showed how a fusion strategy improved the emotion recognition accuracy by fusing the results from EEG and peripheral features at decision level. Our results (see Section 3) show how, contrary to feature level fusion, decision level fusion significantly outperforms the best single modality for arousal classification and does not underperform for valence classification.

In addition to the superior classification performance obtained by multimodal strategy, in the absence of one of the modalities due to temporary problems or artifacts the system can still continue working as single modality emotion detection. The adaptability of the system to remove and add new modalities can be achieved without retraining the classifiers using the DLF. The adaptability and scalability of the DLF strategy gives it another advantage over FLF.

Here, we used two modalities, which are EEG and eye gaze data. The results of the classification over two modalities were fused to obtain the multimodal fusion results. If the classifiers provide confidence measures on their decisions, combining decisions of classifiers can be done using a summation rule. The confidence measure summation fusion was used due to its simplicity and its proven performance for emotion recognition according to [25]. Other decision combination methods including product of confidence measures, decision template fusion, DempsterShafer, Bayesian belief integration [47], weighted sum, and weighted product [48] did not give superior results.

In this paper, the probabilistic outputs of classifiers are used as a measure of confidence. The sum rule is thus defined as follows for a given trial: 

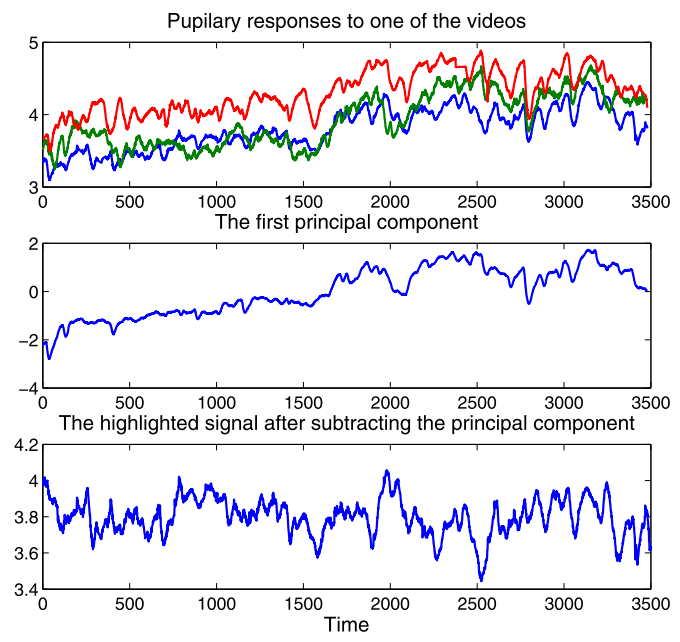

Fig. 7. From top to bottom: In the first plot, there is an example of pupil diameter measures from three different participants in response to one video. The second plot shows the first principal component extracted by PCA from the time series shown in the first plot (the lighting effect). The bottom plot shows the pupil diameter of the blue signal in the first plot after reducing the lighting effect.

$$
g_{a}=\frac{\sum_{q \in Q} P_{q}\left(\omega_{a} \mid x_{i}\right)}{\sum_{a=1}^{K} \sum_{q \in Q} P_{q}\left(\omega_{a} \mid x_{i}\right)}=\sum_{q \in Q} \frac{1}{|Q|} P_{q}\left(\omega_{a} \mid x_{i}\right) .
$$

In (7), $g_{a}$ is the summed confidence interval for affect class $\omega_{a} . Q$ is the ensemble of the classifiers chosen for fusion, $|Q|$ the number of such classifiers, and $P_{q}\left(\omega_{a} \mid x_{i}\right)$ is the posterior probability of having class $\omega_{a}$ the sample is $x_{i}$ according to classifier $q$. The final choice is done by selecting the class $\omega_{a}$ with the highest $g_{a}$. It can be observed that $g_{a}$ can also be viewed as a confidence measure on the class, $\omega_{a}$, given by the fusion of classifiers.

There are two problems in employing SVM classifiers in this fusion scheme. First, they are intrinsically only twoclass classifiers and, second, their output is uncalibrated so that it is not directly usable as a confidence value in case one wants to combine outputs of different classifiers or modalities. To tackle the first problem, the one versus all approach is used where one classifier is trained for each class ( $\mathrm{N}$ classifier to train) and the final choice is done by majority voting. For the second problem, Platt [49] proposes modeling the probability of being in one of the two classes knowing the output value of the SVM by using a sigmoid fit, while $\mathrm{Wu}$ et al. [50] propose a solution to extend this idea to multiple classes. In this study, we used the Matlab libSVM implementation [51] of the Platt and Wu algorithms to obtain the posterior probabilities, $P_{q}\left(\omega_{a} \mid x_{i}\right)$.

\section{EXPERIMENTAL Results}

The experiments were performed in a laboratory environment with controlled temperature and illumination; 24 participants viewed 20 video clips each. Four hundred sixty-seven samples were gathered over a potential data set of $24 \times 20=480$ samples; the 13 missing ones were unavailable due to technical difficulties.

For the pupillary responses to each video, the most significant component was extracted using PCA and then removed from the pupillary time series. In the example

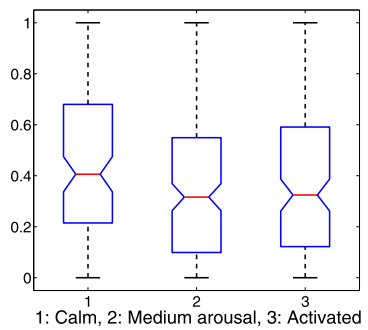

(a) Eye blinking rate, arousal

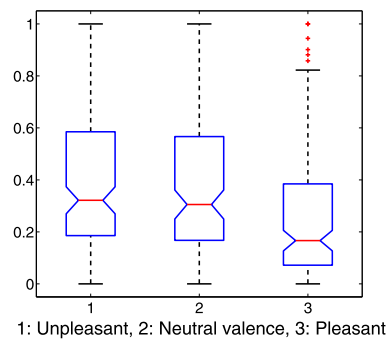

(c) Blink depth, valence

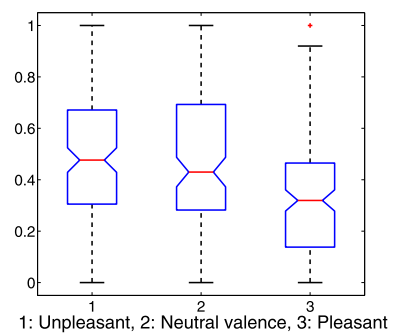

(b) Approach time ration, valence

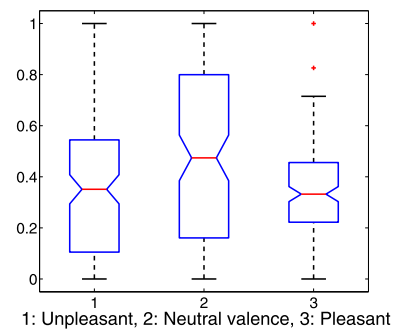

(d) SD of pupil diameter, valence
Fig. 8. Box plots of four different gaze data features in three emotional conditions. (a) Eye blinking rate for arousal classification. (b) Approach time ratio for valence classification. (c) Blink depth, average blink time, for valence classification. (d) STD of pupil diameter for valence classification. One way ANOVA results showed a significant difference between features mean of different classes $(p<0.05)$.

given in Fig. 7, examples of the pupillary responses, extracted pupillary lighting reflex, and the residual component after removing the light reflex are given. The normalized variance or eigen-values of the first component were found to be significantly larger than the rest of the components. The first principal component carried in average more than 50 percent of the variance in the data.

In order to study the discrimination abilities of the eye gaze data features, a one way analysis of variance test was performed on the features. The difference between the mean of features in different arousal or valence categories was found significant $(p<0.05)$. The significance of one way ANOVA shows that there is at least a significant difference between the means of the samples from two classes out of three. The box plots of four features, namely, eye blinking rate, approach rate, maximum blink length, and standard deviation of pupillary responses, are shown in Fig. 8. On average, eye blinking rate was higher in calmer videos (see Fig. 8a). The amount of time participants spent getting closer to the screen is lower for the pleasant category. This shows that they had a tendency to seat more upright while watching more pleasant videos (see Fig. 8b). On the other hand, the maximum blink length or depth is higher for unpleasant videos. This is due to the fact that participants kept their eyes closed for some moments while watching unpleasant videos (see Fig. 8c). Pupillary response's standard deviation is also shown to be higher during neutral scenes (see Fig. 8d).

To find the best discriminative EEG features, the linear discrimination criterion was calculated. This parameter is between class variance divided by within class variance for any given feature (see Table 3 ). For arousal classification, PSD in alpha bands of occipital electrodes was found to be 
TABLE 3

Ten Best EEG Features for Arousal and Valence Classification Based on Linear Discrimination Criterion

\begin{tabular}{l|l|l|l|l|l}
\hline \hline \multicolumn{3}{c}{ Arousal classification } & \multicolumn{3}{c}{ Valence classification } \\
\hline Band & Electrode/s & $\sigma_{b w}^{2} / \sigma_{w n}^{2}$ & Band & Electrode $/ \mathrm{s}$ & $\sigma_{b w}^{2} / \sigma_{w n}^{2}$ \\
\hline Slow $\alpha$ & PO4 & 0.18 & $\beta$ & T8 & 0.08 \\
$\alpha$ & PO4 & 0.17 & $\gamma$ & T8 & 0.08 \\
$\theta$ & PO4 & 0.16 & $\beta$ & T7 & 0.07 \\
Slow $\alpha$ & PO3 & 0.15 & $\gamma$ & T7 & 0.06 \\
$\theta$ & Oz & 0.14 & $\gamma$ & P8 & 0.05 \\
Slow $\alpha$ & O2 & 0.14 & $\gamma$ & P7 & 0.05 \\
Slow $\alpha$ & Oz & 0.14 & $\theta$ & Fp1 & 0.04 \\
$\theta$ & O2 & 0.13 & $\beta$ & CP6 & 0.04 \\
$\theta$ & FC6 & 0.13 & $\beta$ & P8 & 0.04 \\
$\alpha$ & PO3 & 0.13 & $\beta$ & P7 & 0.04 \\
\hline
\end{tabular}

The between class variance to within class variance ratios $\sigma_{b w}^{2} / \sigma_{w n}^{2}$ are also given.

the most discriminant features. In contrast, valence beta and gamma bands of temporal electrodes are more informative. The between class to within class variance ratios are higher for the best arousal EEG features. The higher linear discrimination criterion for best arousal features explains the superior classification rate for arousal dimension (see Table 4).

Regarding the self-reports, we computed the average pairwise Cohen's kappa for keyword based annotations. A fair multirater agreement was found on emotional keywords (nine keywords) with $\kappa=0.32$. The correlation between arousal and valence ratings between participants was also computed. The correlation between arousal and valence ratings given by different participants on nine-point scales were mean $(\rho)=0.45, S D(\rho)=0.25$ and mean $(\rho)=0.73$, $S D(\rho)=0.12$, respectively. Therefore, there was a higher interrater agreement on valence comparing to arousal.

\subsection{Emotion Recognition Feasibility}

Referring to the first research question, the results have shown that it is possible to accurately recognize emotions with a user-independent approach. The classification accuracy measures are summarized in Table 4 . The traditional F-score, which combines precision and recall by their harmonic mean, was also computed for each emotion category to give an overall evaluation of classification performance (8). The F1 score varies between zero and one. The random level is 0.5 for binary classification and balanced classes; values closest to 1 indicate a better performance:

$$
F 1=\frac{2 \times \text { precision } \times \text { recall }}{\text { precision }+ \text { recall }} .
$$

Precision and recall can be only defined for one class; hence, the F1 scores were calculated from the results of one versus all classification schemes for each class separately. As a result, the expectation of F1 scores of a uniform random classifier are calculated and given in Table 4. The classification rate of both three class classifications is defined as the percentage of correctly classified samples.

For the SVM classifier, the size of the kernel, $\gamma$, was selected between $[0.01,10]$, based on the average F1 score using a 20-fold cross validation on the training set. The $C$ parameter that regulates the tradeoff between error minimization and margin maximization is empirically set to 1 .
TABLE 4

The Classification Rate and F1 Scores of Emotion Recognition for Different Modalities

\begin{tabular}{l|l|l|l|l}
\hline \hline \multirow{2}{*}{ Modality } & \multicolumn{2}{|c|}{ Classification rate } & \multicolumn{2}{c}{ Average F1 } \\
\hline dimension & arousal & valence & arousal & valence \\
\hline EEG & $62.1 \%$ & $50.5 \%$ & 0.60 & 0.50 \\
\hline Eye gaze & $71.1 \%$ & $66.6 \%$ & 0.71 & 0.66 \\
\hline $\begin{array}{l}\text { Feature level fusion } \\
\text { (FLF) }\end{array}$ & $66.4 \%$ & $58.4 \%$ & 0.65 & 0.55 \\
\hline $\begin{array}{l}\text { Decision level fusion } \\
\text { (DLF) }\end{array}$ & $76.4 \%$ & $68.5 \%$ & 0.76 & 0.68 \\
\hline $\begin{array}{l}\text { Self-reports with SAM } \\
\text { manikins }\end{array}$ & $55.7 \%$ & $69.4 \%$ & 0.57 & 0.70 \\
\hline Random level & $33.3 \%$ & $33.3 \%$ & 0.36 & 0.40 \\
\hline
\end{tabular}

Classifications were first performed with the goal of recovering the three classes with a leave-one-participantout cross-validation scheme. Regarding the single modality classification of arousal and valence in three classes, we obtained 62.1 and 50.5 percent accuracy from EEG signals and 71.1 and 66.6 percent accuracy from eye gaze data (see Table 4). Although EEG classification results are inferior to the eye gaze data, they are comparable to the state-of-the-art classification rates considering the interparticipant classification scheme [25], [52].

The FLF did not improve the best single modality, gaze data results. However, the modality fusion strategy using the DLF improved the best SVM classification rate for arousal up to 76.4 percent. The DLF did not underperform for valence classification. To test the statistical significance of the classification performance, a paired t-test was used to compare F1 scores of the DLF on one side and the selfreports and the best single modality, eye gaze data, on the other side. The F1 scores from each participant's samples were compared and the improvement over arousal classification comparing to eye gaze data and self-reports were found significant $(p<0.01)$. However, the difference between the eye gaze, DLF, and self-reports F1 scores on valence classification was not found to be statistically significant. The confidence levels of the classification results from the two modalities were added to find the class with the highest summed confidence.

The confusion matrices for each modality show how they performed on each emotion category (Tables 5a, 5b, 5c, 5d, $5 e, 5 f, 5 g, 5 h, 5 i$, and $5 j)$. In these confusion matrices, the row represents the classified label and each column represents the ground truth. Only for the activated category did EEG classification perform as well as gaze data modality. However, the fusion of both, with the exceptions of the neutral valence class, outperformed gaze data results (see Tables 5a, 5b, 5c, 5d and Tables 5f, 5g, 5h, 5i). The DLF outperformed the feature level fusion for all categories except unpleasant (see Tables 5c, 5d, 5h, and 5i).

The use of different stimuli and emotion classes make it difficult to directly compare the results to similar work. Here, we compare the obtained accuracy with the most similar existing studies. Kolodyazhniy et al. [26] used videos extracted from movies in a user independent strategy to classify three classes, namely, neutral, sadness, 
TABLE 5

Confusion Matrices of Different Classification Schemes (Row: Classified Label; Column: Ground Truth)

Arousal

\begin{tabular}{|c|c|c|c|}
\hline & 1 & 2 & 3 \\
\hline 1 & 44 & 15 & 11 \\
\hline 2 & 22 & 111 & 39 \\
\hline 3 & 30 & 60 & 135 \\
\hline
\end{tabular}

(a) EEG

\begin{tabular}{|c|c|c|c|}
\hline & 1 & 2 & 3 \\
\hline 1 & 87 & 56 & 52 \\
\hline 2 & 23 & 52 & 15 \\
\hline 3 & 54 & 31 & 97 \\
\hline
\end{tabular}

(f) EEG

\begin{tabular}{|c|c|c|c|}
\hline & 1 & 2 & 3 \\
\hline 1 & 60 & 7 & 9 \\
\hline 2 & 10 & 136 & 40 \\
\hline 3 & 26 & 43 & 136 \\
\hline
\end{tabular}

(b) Eye gaze data

\begin{tabular}{|c|c|c|c|}
\hline & 1 & 2 & 3 \\
\hline 1 & 108 & 46 & 26 \\
\hline 2 & 36 & 77 & 12 \\
\hline 3 & 20 & 16 & 126 \\
\hline
\end{tabular}

(g) Eye gaze data

\begin{tabular}{|c|c|c|c|}
\hline & 1 & 2 & 3 \\
\hline 1 & 49 & 14 & 10 \\
\hline 2 & 15 & 117 & 31 \\
\hline 3 & 32 & 55 & 144 \\
\hline
\end{tabular}

(c) FLF

\begin{tabular}{|c|c|c|c|}
\hline & 1 & 2 & 3 \\
\hline 1 & 139 & 83 & 56 \\
\hline 2 & 7 & 36 & 10 \\
\hline 3 & 18 & 20 & 98 \\
\hline
\end{tabular}

(h) FLF

\begin{tabular}{|c|c|c|c|}
\hline & 1 & 2 & 3 \\
\hline 1 & 62 & 6 & 8 \\
\hline 2 & 8 & 146 & 28 \\
\hline 3 & 26 & 34 & 149 \\
\hline
\end{tabular}

(d) DLF

\begin{tabular}{|c|c|c|c|}
\hline & 1 & 2 & 3 \\
\hline 1 & 115 & 48 & 22 \\
\hline 2 & 27 & 75 & 12 \\
\hline 3 & 22 & 16 & 130 \\
\hline
\end{tabular}

(i) DLF

\begin{tabular}{|c|c|c|c|}
\hline & 1 & 2 & 3 \\
\hline 1 & 63 & 35 & 11 \\
\hline 2 & 24 & 88 & 65 \\
\hline 3 & 9 & 63 & 109 \\
\hline
\end{tabular}

(e) Self reports

\begin{tabular}{|c|c|c|c|}
\hline & 1 & 2 & 3 \\
\hline 1 & 126 & 40 & 4 \\
\hline 2 & 38 & 91 & 53 \\
\hline 3 & 0 & 8 & 107 \\
\hline
\end{tabular}

(j) Self reports

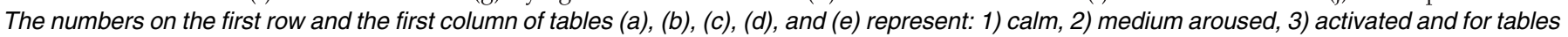

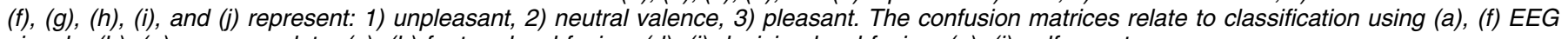
signals, (b), (g) eye gaze data, (c), (h) feature level fusion, (d), (i) decision level fusion, (e), (j) self-reports.

and fear. They obtained 77.5 percent recognition rate from peripheral physiological responses while introducing random startles. Their results on three classes are almost at the same level of our arousal classification performance. In a gaming protocol, Chanel et al. [52] achieved the accuracy of 63 percent in a user-independent approach on the recognition of three classes, namely, boredom, engagement, anxiety. These three classes can be translated to our three arousal levels. Our results are inferior to the ones by Lisetti and Nasoz [14] on six classes. However, we used different modalities and videos and therefore these results are not directly comparable.

\subsection{Emotion Recognition and Self-Reports}

Referring to the second research question, the agreement between participants' self-reports and ground truth is shown in the confusion matrix given in Tables $5 \mathrm{e}$ and $5 \mathrm{j}$. The columns of this table represent how the videos of each class defined by ground truth were individually selfreported. For example, the first column of this table represents how many of the samples which were actually in class one were classified into different classes.

In order to measure the agreement between individual self-reports and the ground truth, the self-reported arousal and valence scores on nine point scale were translated into three levels. These levels were then treated like classified labels and the classification rate was computed. This was done by considering that the goal of each participant is to label a video clip by the correct label, the most common tag. The classification rate for individually self-reported labels was 55.7 percent for arousal, which is inferior to the worst classifier's result. Although, looking at the interannotation agreement, participants found that it easier to self-report pleasantness, the classification rate for valence is not significantly lower than the self-report rate. Therefore, the accuracy of obtained tags via classification is comparable to the individually reported labels.

Fig. 9 summarizes the comparison of different classification strategies showing the F1 scores for each category and on average. Looking at the bars on the rightmost side of the chart, only EEG results are inferior to the explicit self-report agreements using self-assessment manikins (SAM).

\section{Discussion}

In this section, we discuss limitations of the current study and present the open issues. Physiological responses can vary due to nonemotional changes, such as circadian rhythms, ambient temperature, body posture, and other psychophysiological factors such as attention, anticipation, and mental effort [53]. Emotion recognition from bodily responses is therefore confounded by contextual factors. Moreover, like other similar studies [26], the generalization of the results are limited by the videos shown to the participants.

The interannotation agreement for arousal self-reports is lower comparing to keyword-based self-assessments. In a real-case scenario for an explicit tagging system, using words will be easier for an ordinary user and leads to higher between-participant agreement in comparison to arousal and valence reported by self-assessment manikins. However, emotional keywords are difficult to translate and might not exist with the exact same meaning in different languages [32]. Emotion detection can overcome those difficulties with keeping the accuracy at the same level. In this paper, participants were asked to explicitly choose an emotional indicator to form the ground truth. In a real application, with the existence of a reliable user-independent emotion recognition method, the self-reporting phase can be eliminated.

Arousal classes were, on average, detected with higher accuracy using EEG signals comparing to valence labels. This might be due to higher visual and auditory variance of the arousal variant videos comparing to valence variant ones. Exciting scenes usually contain fast movements and loud noises, which manifest themselves both in EEG signals and pupillary responses, whereas the difference between pleasant and unpleasant responses can be hidden in the semantics. The direct bodily responses to different stimuli can increase the variance in responses and improve the

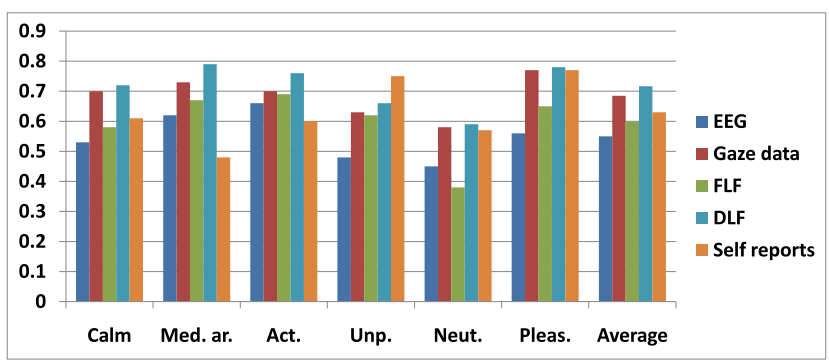

Fig. 9. This bar chart shows the F1 score for classification results of each class from different modalities. 
emotion recognition results. For example, faster changing video induces a different response in occipital cortex activities comparing to a more static video.

The DLF superior classification rate for arousal and its similar performance for valence classification shows that the proposed emotion classification can replace the self-reporting of single participants for detecting popular emotional tags for this data set. These popular emotional tags are defined by the emotions felt by the majority of users watching the same video. After detecting emotional classes, they can be stored with other metadata attached to each video. Emotional labels can be converted to scores for arousal and valence for each video. The emotional scores can then be used as in the image recommendation applications [11] to improve a video recommender's performance. In future, the recognized emotional labels should be added as features to a video recommendation system to study the effect of introducing emotional labels on those systems. This effect can be determined by assessing users' satisfaction from a recommendation or retrieval system with and without emotional information. The emotion detection can be also used indirectly as a tool to detect topical relevance in information retrieval systems [18].

The determination of ground truth is based on the participants' feedback in the online preliminary experiment. In order to measure the agreement between the popular responses of the two separate populations of the preliminary assessments and the experiments, we computed the median valence and arousal reported during the experiment and compared the labels based on the recorded participants' popular response. Only three arousal labels out of 40 labels were changed from activated to medium arousal or vice versa. No valence label has changed when comparing two populations. This is due to the valence dimension's higher interannotator agreement.

This study still has open issues that need to be considered in the future. In a real case scenario, any new user will need to have a few minutes of signals recorded to provide reliable values for feature normalization. The estimation of pupillary response to lighting is an open issue which needs more investigation. Although we assumed that the lighting pupillary responses are similar between participants, there will be a large difference in case of introducing users from a different age group. Therefore, the parameters of a reliable model for pupillary reflex to lighting, such as [43], should be determined before introducing a new user to the system. Alternatively, a real-time lighting effect similar to [29] can be employed to remove the lighting effect. A larger video set and a larger number of participants can be considered to increase the generalization of the developed emotion classifier in response to videos.

In this paper, the length of the experimental session limited the number of videos we could show to each participant. The number of participants in this paper is large and diverse enough comparing to similar studies [1], [25]. However, this population only consisted of young students, which limits the trained algorithm to this particular group. These limitations might worsen the results in the case of introducing a new genre of video which was not present in the current video set. To generalize and train such a system, the recruited participants should be as close as possible to the target audience of the video retrieval or recommendation systems.

Emotions can co-occur and/or last for very short moments. This puts using a single emotional label for a video clip under question. In order to address this issue, self-reporting should include the possibility to indicate different emotions and their degree of strength. This is possible by using questionnaires such as positive and negative affect schedule (PANAS) [54] or the Geneva emotion wheel [9]. However, these emotional self-reporting methods are more complex and make the experiment longer. In future, multiple or co-occurring emotions should be assessed using a more sophisticated self-reporting tool.

\section{Conclusions}

This paper showed the performance of an interparticipant emotion recognition tagging approach using participants' EEG signals, gaze distance and pupillary response as affective feedbacks. The feasibility of an approach to recognize emotion in response to videos is shown. Although the results were based on a fairly small video data set due to experimental limitations, the promising accuracy can be scalable to more samples from a larger population. The improved performance using multimodal fusion techniques leads to the conclusion that by adding other modalities, such as facial expressions, accuracy as well as robustness should further improve. Results from our previous studies [3] showed that there is a significant difference between peoples' emotional self-assessments in response to videos. However, there usually exists one most popular emotional tag for which there is significant agreement in a population. This "most popular emotion" has been shown to be detectable with monitoring users' bodily responses. Moreover, the population tags give the retrieval system a higher chance of success in a given population. We have shown that it is possible to design an accurate and user-independent classification protocol to recognize emotions from pupillary reflex, EEG signals in response to video content. Moreover, we have shown that for the video data set utilized, the nonverbal affective cues can replace affective self-report with comparable emotion recognition performance and no requisite of direct user inputs. We can thus answer positively to our two research questions.

\section{ACKNOWLEDGMENTS}

The work of Mohammad Soleymani and Thierry Pun was supported in part by the Swiss National Science Foundation and in part by the European Community's Seventh Framework Programme (FP7/2007-2011) under grant agreement Petamedia no. 216444. The data acquisition part of this work was supported by the European Research Council under the ERC Starting Grant agreement no. ERC-2007-StG-203143 (MAHNOB). The work of Maja Pantic is supported in part by the European Community's 7th Framework Programme (FP7/2007-2013) under grant agreement no. 231287 (SSPNet). The authors would like to thank Dr. Jeroen Lichtenauer and Jozef Doboš (Imperial College London), Prof. Didier Grandjean, and Dr. Guillaume Chanel (University of Geneva) for their valuable scientific comments and technical support during the experiments. 


\section{REFERENCES}

[1] J. Kim and E. André, "Emotion Recognition Based on Physiological Changes in Music Listening," IEEE Trans. Pattern Analysis and Machine Intelligence, vol. 30, no. 12, pp. 2067-2083, Dec. 2008.

[2] J. Rottenberg, R.D. Ray, and J.J. Gross, Emotion Elicitation Using Films, pp. 9-28, series in affective science. Oxford Univ. Press, 2007.

[3] M. Soleymani, G. Chanel, J.J.M. Kierkels, and T. Pun, “Affective Characterization of Movie Scenes Based on Content Analysis and Physiological Changes," Int'l J. Semantic Computing, vol. 3, no. 2, pp. 235-254, June 2009.

[4] R.W. Picard and S.B. Daily, "Evaluating Affective Interactions: Alternatives to Asking What Users Feel," Proc. CHI Workshop Evaluating Affective Interfaces: Innovative Approaches, 2005.

[5] K.R. Scherer, "Studying the Emotion-Antecedent Appraisal Process: An Expert System Approach," Cognition \& Emotion, vol. 7, no. 3, pp. 325-355, 1993.

[6] A. Ortony, G.L. Clore, and A. Collins, The Cognitive Structure of Emotions. Cambridge Univ. Press, July 1988.

[7] D. Sander, D. Grandjean, and K.R. Scherer, "A Systems Approach to Appraisal Mechanisms in Emotion," Neural Networks, vol. 18, no. 4, pp. 317-352, May 2005.

[8] W. Wirth and H. Schramm, "Media and Emotions," Comm. Research Trends, vol. 24, no. 3, pp. 3-39, 2005.

[9] K.R. Scherer, "What Are Emotions? And How Can They Be Measured?" Social Science Information, vol. 44, no. 4, pp. 695-729, Dec. 2005.

[10] A. Hanjalic and L.-Q. Xu, "Affective Video Content Representation and Modeling," IEEE Trans. Multimedia, vol. 7, no. 1, pp. 143154, http://dx.doi.org/10.1109/TMM.2004.840618, Feb. 2005.

[11] M. Tkalčič, U. Burnik, and A. Košir, "Using Affective Parameters in a Content-Based Recommender System for Images," User Modeling and User-Adapted Interaction, vol. 20, pp. 279-311, Oct. 2010.

[12] M.K. Shan, F.F. Kuo, M.F. Chiang, and S.Y. Lee, "Emotion-Based Music Recommendation by Affinity Discovery from Film Music," Expert Systems Applications, vol. 36, no. 4, pp. 7666-7674, Sept. 2009.

[13] H. Joho, J.M. Jose, R. Valenti, and N. Sebe, "Exploiting Facial Expressions for Affective Video Summarisation," Proc. ACM Int'l Conf. Image and Video Retrieval, 2009.

[14] C.L. Lisetti and F. Nasoz, "Using Noninvasive Wearable Computers to Recognize Human Emotions from Physiological Signals," EURASIP J. Applied Signal Processing, vol. 2004, no. 1, pp. 16721687, Jan. 2004

[15] K. Takahashi, "Remarks on Emotion Recognition from BioPotential Signals," Proc. Second Int'l Conf. Autonomous Robots and Agents, 2005.

[16] S. Koelstra, A. Yazdani, M. Soleymani, C. Mühl, J.-S. Lee, A. Nijholt, T. Pun, T. Ebrahimi, and I. Patras, "Single Trial Classification of EEG and Peripheral Physiological Signals for Recognition of Emotions Induced by Music Videos," Proc. Int'l Conf. Brain Informatics, Y. Yao, R. Sun, T. Poggio, J. Liu, N. Zhong, and J. Huang, eds., ch. 9, pp. 89-100, 2010.

[17] H. Joho, J. Staiano, N. Sebe, and J. Jose, "Looking at the Viewer: Analysing Facial Activity to Detect Personal Highlights of Multimedia Contents," Multimedia Tools and Applications, pp. 1-19, Oct. 2010.

[18] I. Arapakis, I. Konstas, and J.M. Jose, “Using Facial Expressions and Peripheral Physiological Signals as Implicit Indicators of Topical Relevance," Proc. 17th ACM Int'l Conf. Multimedia, pp. 461-470, 2009.

[19] G. Irie, T. Satou, A. Kojima, T. Yamasaki, and K. Aizawa, "Affective Audio-Visual Words and Latent Topic Driving Model for Realizing Movie Affective Scene Classification," IEEE Trans. Multimedia, vol. 12, no. 6, pp. 523-535, Oct. 2010.

[20] M. Soleymani, J.J.M. Kierkels, G. Chanel, and T. Pun, "A Bayesian Framework for Video Affective Representation," Proc. Int'l Conf. Affective Computing and Intelligent Interaction, pp. 1-7, Sept. 2009.

[21] P. Lang, M. Bradley, and B. Cuthbert, "International Affective Picture System (IAPS): Affective Ratings of Pictures and Instruction Manual," Technical Report A-8, Univ. of Florida, Gainesville, 2008.

[22] P.J. Lang, M.K. Greenwald, M.M. Bradley, and A.O. Hamm, "Looking at Pictures: Affective, Facial, Visceral, and Behavioral Reactions," Psychophysiology, vol. 30, no. 3, pp. 261-273, 1993.

[23] J. Wang and Y. Gong, "Recognition of Multiple Drivers' Emotional State," Proc. 19th Int'l Conf. Pattern Recognition, 2008.
[24] J.A. Healey, "Wearable and Automotive Systems for Affect Recognition from Physiology," PhD dissertation, MIT, 2000.

[25] G. Chanel, J.J.M. Kierkels, M. Soleymani, and T. Pun, "Short-Term Emotion Assessment in a Recall Paradigm," Int'l J. HumanComputer Studies, vol. 67, no. 8, pp. 607-627, Aug. 2009.

[26] V. Kolodyazhniy, S.D. Kreibig, J.J. Gross, W.T. Roth, and F.H. Wilhelm, "An Affective Computing Approach to Physiological Emotion Specificity: Toward Subject-Independent and StimulusIndependent Classification of Film-Induced Emotions," Psychophysiology, vol. 7, no. 48, pp. 908-922, 2011.

[27] M.M. Bradley, L. Miccoli, M.A. Escrig, and P.J. Lang, "The Pupil as a Measure of Emotional Arousal and Autonomic Activation," Psychophysiology, vol. 45, no. 4, pp. 602-607, July 2008.

[28] T. Partala and V. Surakka, "Pupil Size Variation as an Indication of Affective Processing," Int'l J. Human-Computer Studies, vol. 59, nos. 1/2, pp. 185-198, 2003.

[29] Y. Gao, A. Barreto, and M. Adjouadi, "Monitoring and Processing of the Pupil Diameter Signal for Affective Assessment of a Computer User," Proc. 13th Int'l Conf. Human-Computer Interaction. Part I: New Trends, pp. 49-58, 2009.

[30] J.J.M. Kierkels, M. Soleymani, and T. Pun, "Queries and Tags in Affect-Based Multimedia Retrieval," Proc. IEEE Int'l Conf. Multimedia and Expo, pp. 1436-1439, 2009.

[31] M. Pantic and A. Vinciarelli, "Implicit Human-Centered Tagging," IEEE Signal Processing Magazine, vol. 26, no. 6, pp. 173-180, Nov. 2009.

[32] J.A. Russell, "Culture and the Categorization of Emotions," Psychological Bull., vol. 110, no. 3, pp. 426-450, 1991.

[33] J.A. Russell and A. Mehrabian, "Evidence for a Three-Factor Theory of Emotions," J. Research in Personality, vol. 11, no. 3, pp. 273-294, Sept. 1977.

[34] M. Soleymani, J. Davis, and T. Pun, "A Collaborative Personalized Affective Video Retrieval System," Proc. Third Int'l Conf. Affective Computing and Intelligent Interaction and Workshops, Sept. 2009.

[35] A. Schaefer, F. Nils, X. Sanchez, and P. Philippot, "Assessing the Effectiveness of a Large Database of Emotion-Eliciting Films: A New Tool for Emotion Researchers," Cognition \& Emotion, vol. 24, no. 7, pp. 1153-1172, 2010.

[36] J. Lichtenauer, M. Valstar, J. Shen, and M. Pantic, "Cost-Effective Solution to Synchronized Audio-Visual Capture Using Multiple Sensors," Proc. Sixth IEEE Int'l Conf. Advanced Video and Signal Based Surveillance, pp. 324-329, 2009.

[37] R. Adolphs, D. Tranel, and A.R. Damasio, "Dissociable Neural Systems for Recognizing Emotions," Brain and Cognition, vol. 52, no. 1, pp. 61-69, June 2003.

[38] A.R. Damasio, T.J. Grabowski, A. Bechara, H. Damasio, L.L.B. Ponto, J. Parvizi, and R.D. Hichwa, "Subcortical and Cortical Brain Activity during the Feeling of Self-Generated Emotions," Nature Neuroscience, vol. 3, no. 10, pp. 1049-1056, Oct. 2000.

[39] R.J. Davidson, "Affective Neuroscience and Psychophysiology: Toward a Synthesis," Psychophysiology, vol. 40, no. 5, pp. 655-665, Sept. 2003.

[40] L.I. Aftanas, N.V. Reva, A.A. Varlamov, S.V. Pavlov, and V.P. Makhnev, "Analysis of Evoked EEG Synchronization and Desynchronization in Conditions of Emotional Activation in Humans: Temporal and Topographic Characteristics," Neuroscience and Behavioral Physiology, vol. 34, no. 8, pp. 859-867, Oct. 2004.

[41] P.D. Welch, "The Use of Fast Fourier Transform for the Estimation of Power Spectra: A Method Based on Time Averaging over Short, Modified Periodograms," IEEE Trans. Audio and Electroacoustics, vol. 15, no. 2, pp. 70-73, June 1967.

[42] S.K. Sutton and R.J. Davidson, "Prefrontal Brain Asymmetry: A Biological Substrate of the Behavioral Approach and Inhibition Systems," Psychological Science, vol. 8, no. 3, pp. 204-210, 1997.

[43] V.F. Pamplona, M.M. Oliveira, and G.V.G. Baranoski, "Photorealistic Models for Pupil Light Reflex and Iridal Pattern Deformation," ACM Trans. Graphics, vol. 28, no. 4, pp. 1-12, 2009.

[44] A. Longtin and J. Milton, "Modelling Autonomous Oscillations in the Human Pupil Light Reflex Using Nonlinear Delay-Differential Equations," Bull. Math. Biology, vol. 51, no. 5, pp. 605-624, Sept. 1989.

[45] H. Bouma and L.C.J. Baghuis, "Hippus of the Pupil: Periods of Slow Oscillations of Unknown Origin," Vision Research, vol. 11, no. 11, pp. 1345-1351, 1971.

[46] F.H. Kanfer, "Verbal Rate, Eyeblink, and Content in Structured Psychiatric Interviews," J. Abnormal and Social Psychology, vol. 61, no. 3, pp. 341-347, 1960. 
[47] D. Ruta and B. Gabrys, "An Overview of Classifier Fusion Methods," Computing and Information Systems, vol. 7, no. 1, pp. 110, 2000.

[48] L.I. Kuncheva, Combining Pattern Classifiers: Methods and Algorithms. Wiley-Interscience, July 2004.

[49] J.C. Platt, "Probabilities for SV Machines," Advances in Large Margin Classifier, pp. 61-74, MIT Press, 2000.

[50] T.F. Wu, C.J. Lin, and R.C. Weng, "Probability Estimates for MultiClass Classification by Pairwise Coupling," J. Machine Learning Research, vol. 5, pp. 975-1005, 2004.

[51] C. Chang and C. Lin, "LIBSVM: A Library for Support Vector Machines," Science, vol. 2, pp. 1-39, 2001.

[52] G. Chanel, C. Rebetez, M. Bétrancourt, and T. Pun, "Emotion Assessment from Physiological Signals for Adaptation of Game Difficulty," IEEE Trans. Systems, Man, and Cybernetics, Part A: Systems and Humans, vol. 41, no. 6, pp. 1052-1063, Nov. 2011.

[53] S.D. Kreibig, G. Schaefer, and T. Brosch, Psychophysiological Response Patterning in Emotion: Implications for Affective Computing. ch. 2.4, pp. 105-130, Oxford Univ. Press, 2010.

[54] D. Watson, L.A. Clark, and A. Tellegen, "Development and Validation of Brief Measures of Positive and Negative Affect: The PANAS Scales," J. Personality and Social Psychology, vol. 54, no. 6, pp. 1063-1070, June 1988.

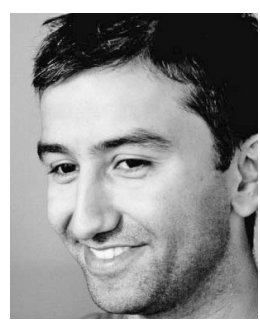

Mohammad Soleymani received both the BSc and MSc degrees from the Department of Electrical and Computer Engineering, University of Tehran, Iran, in 2003 and 2006, respectively. $\mathrm{He}$ is currently working toward the doctoral degree and is a research assistant in the Computer Vision and Multimedia Laboratory, Computer Science Department, University of Geneva, Switzerland. His research interests include affective computing and multimedia information retrieval. He has been coorganizing the MediaEval multimedia benchmarking initiative since 2010. He is a member of the IEEE and the HUMAINE association.

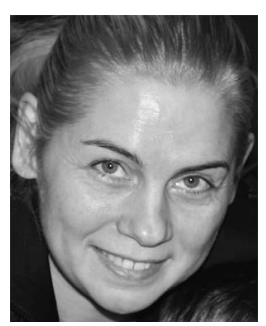

Maja Pantic is a professor in affective and behavioral computing in the Department of Computing, Imperial College London, United Kingdom, and Department of Computer Science, University of Twente, The Netherlands. She is one of the world's leading experts in the research on machine understanding of human behavior, including vision-based detection, tracking, and analysis of human behavioral cues like facial expressions and body gestures, and multimodal human affect/mental state understanding. She has published more than 100 technical papers in these areas of research. In 2008, for her research on Machine Analysis of Human Naturalistic Behavior (MAHNOB), she received the European Research Council Starting Grant as one of 2 percent of the best young scientists in any research field in Europe. In 2011, she was awarded the Roger Needham Award, made annually for a distinguished research contribution in computer science by a United Kingdom-based researcher within 10 years of their $\mathrm{PhD}$. She currently serves as the editor in chief of Image and Vision Computing Journal and as an associate editor for the IEEE Transactions on Systems, Man, and Cybernetics Part B (TSMC-B). She has also served as the general chair for several conferences and symposia, including IEEE FG 2008 and IEEE ACII 2009. She is a fellow of the IEEE.

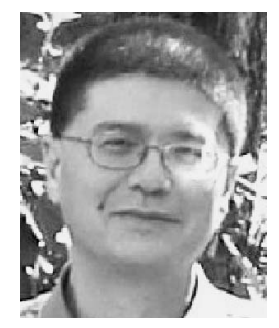

Thierry Pun received the electrical engineer diploma in 1979. He received the PhD degree in image processing for the development of a visual prosthesis for the blind in 1982 from the Swiss Federal Institute of Technology, Lausanne. He is head of the Computer Vision and Multimedia Laboratory, Computer Science Department, University of Geneva, Switzerland. He was a visiting fellow from 1982 to 1985 at the National Institutes of Health, Bethesda, Maryland. After being a CERN fellow from 1985 to 1986 in Geneva, Switzerland, he joined the University of Geneva in 1986, where he is currently a full professor in the Computer Science Department. He has authored or coauthored about 300 full papers as well as eight patents. His current research interests, related to affective computing and multimodal interaction, concern: physiological signals analysis for emotion assessment and braincomputer interaction, multimodal interfaces for blind users, data hiding, multimedia information retrieval systems. $\mathrm{He}$ is a member of the IEEE.

For more information on this or any other computing topic, please visit our Digital Library at www.computer.org/publications/dlib. 